\title{
Micro-EDM numerical simulation and experimental validation
}

\author{
Samuel Bigot ${ }^{1}$, Jean-Philippe Pernot ${ }^{2}$, Anthony Surleraux ${ }^{1}$, Ahmed Elkaseer $^{1}$ \\ ${ }^{1}$ Manufacturing Engineering Centre, Cardiff University, Cardiff, UK, e-mail: bigots@cf.ac.uk \\ ${ }^{2}$ Arts et Métiers ParisTech, LSIS - UMR CNRS 7296, Aix-en-Provence, France
}

\begin{abstract}
This paper introduces a new method for simulating the micro-EDM process in order to predict tool wear. The tool and workpiece are defined by NURBS surfaces whose shapes result from an iterative crater-by-crater deformation technique driven by physical parameters. The simulation method is validated through a comparison with experimental data. Different simulations are presented with an increase in computation accuracy in order to study its influence on the results and their deviation from expected values.
\end{abstract}

Keywords: Micro electrical discharge machining, crater, wear, simulation, NURBS surface deformation.

\section{Introduction}

Electrical Discharge Machining (EDM) is a manufacturing process that consists in removing parts of a material with electrical discharges and is characterized by its ability to machine any conductive material regardless of its hardness.

Although various forms of EDM exist all of them share the same concept: two electrodes (the tool and the workpiece) are separated by a dielectric fluid. Both electrodes are submitted to an electrical current and as the gap between the electrodes diminishes, the intensity between them increases until it reaches what is called the dielectric breakdown voltage. At this point, the dielectric cannot act as an insulator anymore and allows current to flow from one electrode to another leading to the apparition of a plasma channel. The plasma's temperature ranges from 8,000 to $12,000^{\circ} \mathrm{C}$ and in some cases can reach up to $20,000^{\circ} \mathrm{C}$ [1]. This leads to evaporation and melting of both the tool and the workpiece. When the current is stopped, the dielectric fluid rushes back where the plasma stood and evacuates resulting debris.

Micro-EDM ( $\mu$ EDM) shares the same underlying concepts of EDM. It simply tackles with dimensions in the order of the micron.

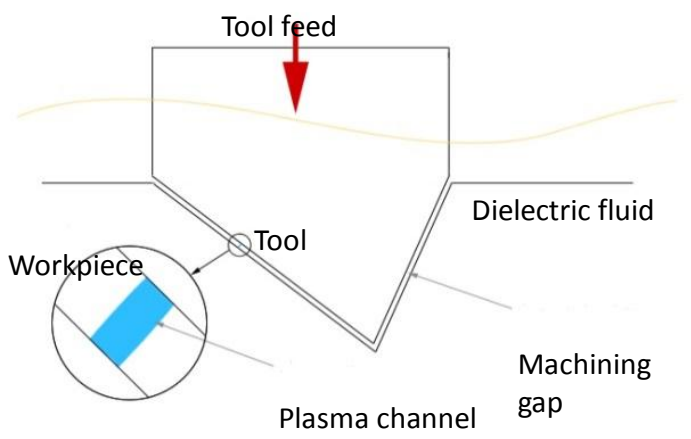

Fig. 1. Principle of EDM
However, while the process setup is modified in order to reduce its effect, tool wear often becomes the main factor of imprecision. When using conventional machining strategies, the electrodes' shapes quickly deviates from the original ones. Thus, as of now $\mu \mathrm{EDM}$ milling is the preferred machining strategy for the fact that proven methods [2] exist to mitigate the influence of tool wear on the final result, while for similar applications die-sinking $\mu$ EDM may require a dozen or more tools before obtaining the desired geometrical tolerances.

Being able to predict the tool wear more accurately would enable us to design more efficient machining strategies, in particular for die sinking EDM where using extra volumes on specific parts of a tool electrode could compensate partially for the wear and drastically reduce the number of electrodes required. To achieve this, a better theoretical understanding of the wear phenomena is required. Thus, in a previous work [3] it was proposed to develop a new modelling framework to facilitate the development and validation of theoretical models of the wear and a new simulation method was introduced that uses two Non-Uniform Rational BSplines (NURBS) surfaces to simulate the evolution of the resulting tool and workpiece shapes using a crater-by-crater iterative deformation technique. In this paper, a new version of this simulation method (section 2) is described and its performance is compared with the results obtained from several EDM experiments (section 3 ).

\section{Micro-EDM simulation}

\subsection{Overview of the new simulation process}

In the proposed approach, both the geometry of the tool and the geometry of the workpiece are defined by means of NURBS patches (see [5] for a complete description of the underlying mathematical 
models). To allow the insertion of thousands of craters, the surfaces of the tool $\boldsymbol{S}_{t}$ and workpiece $\boldsymbol{S}_{w}$ are heavily refined using the Boehm's knot insertion algorithm [5]. As a result the surfaces' control points will be a lot closer to it hence local control is significantly increased. At each step of the insertion process the location of each crater (one on each electrode) is determined while identifying the shortest distance between the tool and the workpiece since it is considered that the electrical spark will happen on the less resistive path, i.e. the shortest one.

Minimum distance computations are done using an optimization method known as particle swarm optimization (PSO) which is a simple numerical optimizer that does not require the use of the gradient of the objective function [4]. A crater is then inserted in each of those locations by moving the surrounding control points. If the computed minimum distance exceeds the value of the minimum distance required for a spark to appear (known in EDM as the gap distance $\mathrm{M}_{\mathrm{g}}$ ) then the tool is moved down along the $\boldsymbol{z}$ axis with an increment of $\Delta_{\mathrm{z}}$. Otherwise, if the computed minimum distance is smaller than $\mathrm{M}_{\mathrm{g}}$ then the PSO algorithm returns four values $\left(u_{t}, v_{t}\right)$ and $\left(u_{w}, v_{w}\right)$ corresponding to the parametric coordinates of the craters' centres respectively on the tool (subscript $t$ ) and workpiece (subscript $w$ ). The algorithm then moves the control points located in the surrounding of the craters' centres so that two craters of volumes $V_{t}$ and $V_{w}$ are inserted into the tool and workpiece. The deformation technique is similar to surface warping [5] considered as a geometric deformation technique [6]. The process ends when the desired depth $D_{t}$ is met. The overall algorithm can be described in pseudo-code form as follows:

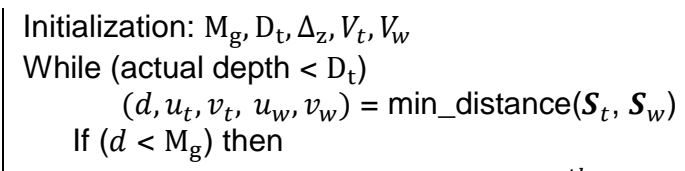

Entering the loop for inserting the $k^{\text {th }}$ craters on both surfaces $\boldsymbol{S}_{i}$ with $i \in\{t, w\}$ :

Compute warping vectors $\omega_{i}^{[k]}$

Compute spheres centres' positions $C_{i}^{[k]}$ Identify the $N_{i}^{[k]}$ control points of $\boldsymbol{S}_{i}$ to be moved in the $\omega_{i}^{[k]}$ direction

For each control point $\boldsymbol{P}_{i, j}^{[k]}, j \in\left\{1, \ldots, N_{i}^{[k]}\right\}$ and $i \in\{t, w\}$ :

Compute the deviation $r_{i, j}^{[k]}$ from $\boldsymbol{\omega}_{i}^{[k]}$

Compute warping value $f_{i}^{[k]}\left(r_{i, j}^{[k]}\right)$

Move control point along $\boldsymbol{\omega}_{i}^{[k]}$ so that its new position $\widetilde{\boldsymbol{P}}_{i, j}^{[k]}$ is defined by:

$\widetilde{\boldsymbol{P}}_{i, j}^{[k]}=\boldsymbol{P}_{i, j}^{[k]}+f_{i}^{[k]}\left(r_{i, j}^{[k]}\right) \cdot \boldsymbol{\omega}_{i}^{[k]}$

$$
\text { End for }
$$

Else

End if

move_tool_down $\left(\boldsymbol{S}_{t}, \Delta_{\mathrm{z}}\right)$

End while
The different steps of the crater insertion process are further detailed in the next subsection.

\subsection{Volume computation and crater insertion}

For a given depth of the tool, if the minimum distance is smaller than the gap distance, the craters insertion process starts. One crater will be inserted on each surface $\boldsymbol{S}_{i}(i \in\{t, w\})$ and centred on $\boldsymbol{S}_{i}\left(u_{i}, v_{i}\right)$. For sake of clarity, the superscript $[k]$ has not been put on the parametric coordinates $u_{i}$ and $v_{i}$ even if these values refer to the $k^{\text {th }}$ craters (one on each surface).

First, to identify the displacement directions, the two warping unit vectors are computed as follows:

$$
\boldsymbol{\omega}_{i}^{[k]}=\operatorname{sg}(i) \cdot \frac{\boldsymbol{S}_{t}\left(u_{t}, v_{t}\right)-\boldsymbol{S}_{w}\left(u_{w}, v_{w}\right)}{\left\|\boldsymbol{S}_{t}\left(u_{t}, v_{t}\right)-\boldsymbol{S}_{w}\left(u_{w}, v_{w}\right)\right\|}
$$

with $\operatorname{sg}(i)=\left\{\begin{array}{c}1 \text { for } i=t \\ -1 \text { for } i=w\end{array}\right.$

Figure 2 represents a two-dimensional version of the process after having found the minimum distance. The case considered here is where the workpiece is to be deformed.

The next step consists in identifying which control points need to be moved in the surrounding of the two points $S_{i}\left(u_{i}, v_{i}\right)$. Actually, each electrical spark transfers a certain amount of energy to the tool, the workpiece and the dielectric fluid. Here, it is considered that the amount of energy brought to each element is the same at each spark. As such, it is desirable to remove the same volumes $V_{t}$ (tool) and $V_{w}$ (workpiece) when simulating the insertion of all the craters. These volumes are experimentally obtained by measuring the mean radius $R_{i}^{m}$ and mean depth $D_{i}^{m}$ of actual craters. Then, considering that the craters are domes, the crater volumes are computed using the following formula:

$$
V_{i}=\pi \cdot \frac{R_{i}^{m}}{6}\left[3 R_{i}^{m^{2}}+D_{i}^{m^{2}}\right], i \in\{t, w\}
$$

From these volumes and domes, the support spheres can be identified, i.e. the spheres of radii $R_{i}$ equal to the dome's radius. As explain, these two radii remain constant for the two surfaces for the crater-by-crater simulation.

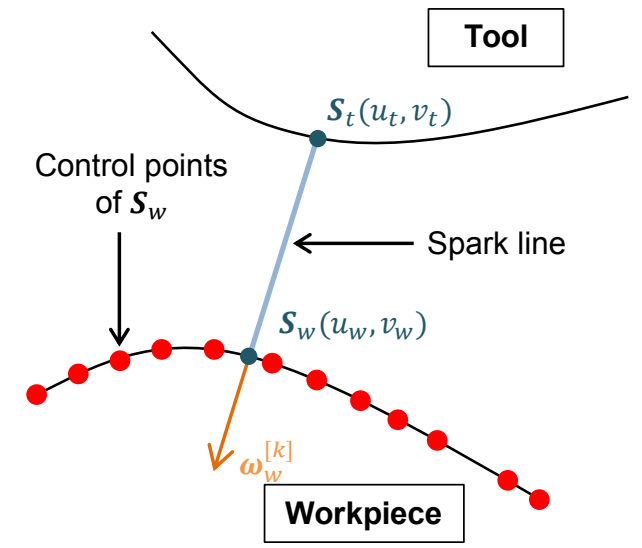

Fig. 2. Warping vector definition for a crater to appear on the workpiece. 


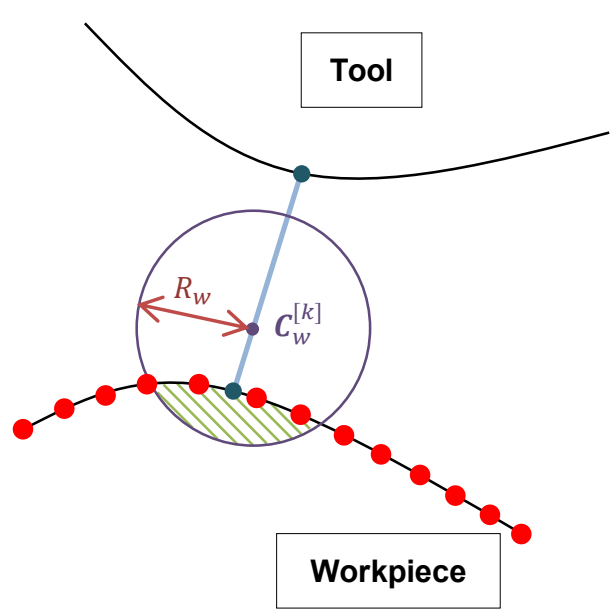

Fig. 3. Definition of the support sphere centred in $\boldsymbol{C}_{w}^{[k]}$.

Once the radii of the two spheres identified, the location of the spheres' centres has to be computed (one for the tool and one for the workpiece). As illustrated on figure 3 , the centre of the sphere lies on the spark line. Its exact position depends on the volume $V_{i}$ that needs to be removed. In order to find the location, an iterative dichotomy method (also known as binary search or bisection method) is used. At each step, the intersecting volume (the hashed part of figure 3) between the sphere and the surface is computed. If the volume obtained is smaller than the target $V_{i}$ the sphere is moved towards the surface and if it is bigger it is moved away from it. The process carries on until the obtained volume falls within a specific tolerance $T_{v}$. Once the $C_{i}{ }^{[k]}$ adequate positions are found, it is possible to determine the $N_{i}{ }^{k k]}$ control points of the two $\boldsymbol{S}_{i}$ surfaces that need to be moved. This is done by computing for each control point the distance that separates them from the centre of the sphere. If the distance is smaller than the radius of the sphere, the control point is added to the list of points to be displaced. At the end, two lists of control points are obtained.

In order to displace the control points to mimic the shape of a sphere, a reference is needed. Let $\Pi_{i}[k]$ be the plane that includes the centre of the sphere $C_{i}[k]$ and that has $\boldsymbol{\omega}_{i}{ }^{[k]}$ as normal vector. Then, for all the control points, $\boldsymbol{P}_{i, j}[k], j \in\left\{1, \ldots, N_{i}[k]\right)$ and $i \in\{t, w\}$, the new position are computed as follows (figure 4):

$$
\begin{gathered}
\widetilde{\boldsymbol{P}}_{i, j}^{[k]}=\boldsymbol{P}_{i, j}^{[k]}+f_{i, j}^{[k]}\left(r_{i, j}^{[k]}\right) \cdot \boldsymbol{\omega}_{i}^{[k]} \\
\text { with } f_{i, j}^{[k]}\left(r_{i, j}^{[k]} \boldsymbol{F}\right)=\sqrt{R_{i}^{2}-r_{i, j}^{[k]} \Re^{2}}-\boldsymbol{\omega}_{i}^{[k]} \cdot\left(\boldsymbol{P}_{i, j}^{[k]}-\right. \\
\left.\boldsymbol{\pi}_{i, j}^{[k]}\right) \text { and } r_{i, j}^{[k]}=\left\|\boldsymbol{\pi}_{i, j}^{[k]}-\boldsymbol{C}_{i}^{[k]}\right\|, \boldsymbol{\pi}_{i, j}^{[k]} \text { being the } \\
\text { projection of } \boldsymbol{P}_{i, j}^{[k]} \text { on the plane } \Pi_{i}^{[k]}
\end{gathered}
$$

This process is repeated iteratively until no more craters can be inserted for the actual depth. Then, the tool is moved down along the $\boldsymbol{z}$ axis with an increment of $\Delta_{\mathrm{z}}$ and the craters insertion process starts again.

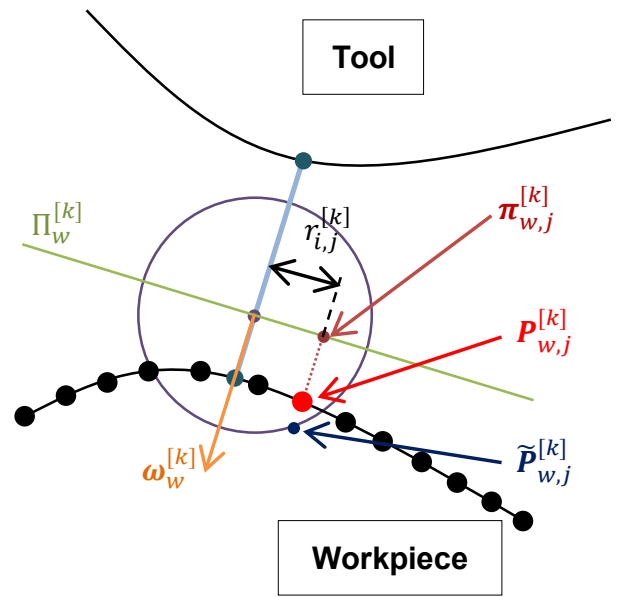

Fig. 4. Plane $\Pi_{w}^{[k]}$ Definition and a control point's projection.

\section{Experimental validation}

As an initial evaluation of the new simulation process, two simple experiments were conducted on a Sarix SX-200 $\mu$ EDM machine equipped with a wire dressing unit. A tungsten carbide rod, with a nominal diameter of $290 \mu \mathrm{m}$ was used as tool electrode. Ultra Fine Grained aluminium (Al1070) with an average grain size of $0.6 \mu \mathrm{m}$ was chosen as workpiece material to minimise material's inhomogeneity while aiming at improving $\mu E D M$ predictability, as suggested in [7].

Using the wire dressing unit, the tip of the tool electrode was machined flat for experiment 1 while for experiment 2 a curved shape was introduced (figure 5a). The electrodes were then used to erode the workpiece down to a $50 \mu \mathrm{m}$ depth. Machining parameters and results are shown in tables 1 and 2.Three simulations of Experiment 1 were then performed using different tolerances $T_{v}$ to assess the influence of the computational precision on roughness results (Table 3 ). The target volume $V_{i}$ expected to be removed per crater was $279 \mu^{3}$.

Table 1

Machining parameters

\begin{tabular}{l|c|c}
\hline Experiment & 1 & 2 \\
\hline Energy level (index) & 300 & 13 \\
Voltage (V) & 60 & 60 \\
Current (index) & 20 & 20 \\
Time on (ms) & 5 & 5 \\
\hline
\end{tabular}

Table 2

Experimental results

\begin{tabular}{l|c|c}
\hline Experiment & 1 & 2 \\
\hline Hole depth $(\mu \mathrm{m})$ & 50.8 & 50.2 \\
Tool vertical wear $(\mu \mathrm{m})$ & 12.5 & 11.3 \\
Roughness, $\mathrm{Ra}(\mu \mathrm{m})$ & 1.27 & 0.82 \\
Workpiece crater diameter $(\mu \mathrm{m})$ & 15 & 3 \\
Workpiece crater depth $(\mu \mathrm{m})$ & 3 & 1 \\
\hline
\end{tabular}


Table 3

Effect of volume removal precision on roughness

\begin{tabular}{l|rrr}
\hline \multicolumn{1}{c|}{ Tolerance $(\%)$} & 10 & 5 & 1 \\
\hline Volume removed $\left(\mu \mathrm{m}^{3}\right)$ & 172058 & 169978 & 167586 \\
$\begin{array}{l}\text { Average volume }\left(\mu \mathrm{m}^{3}\right) \\
\text { removed per crater }\end{array}$ & 286,76 & 283,30 & 279,31 \\
Roughness, $\mathrm{Ra}(\mu \mathrm{m})$ & 1,87 & 1,88 & 1,43 \\
\hline
\end{tabular}

Table 4

Experiment and simulation results

\begin{tabular}{l|l}
\hline Experimental tool vertical wear $(\mu \mathrm{m})$ & 11.3 \\
Simulated tool vertical wear $(\mu \mathrm{m})$ & 11.2 \\
Experimental roughness, $\mathrm{Ra}(\mu \mathrm{m})$ & 0.82 \\
Simulated roughness $(\mu \mathrm{m})$ & 0.87 \\
\hline
\end{tabular}

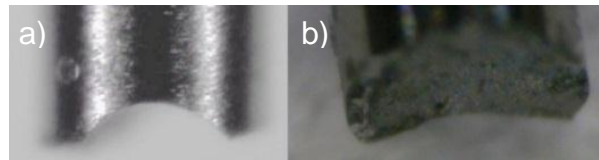

Fig.5. Experimental tool : a) before, b) after machining a)

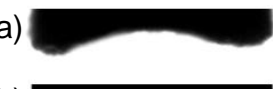

b)

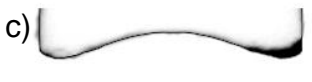

Fig.6. Tool profiles. a) Experimentation b) Simulation c)

Profiles differences. black: simulation extra volume.

Table 3, clearly shows the significant influence of the tolerance level on the simulated roughness.

Following this, one simulation was performed for Experiment 2 to assess agreements in terms of geometry deformation due to tool wear, as well as achieved roughness. Based on the previous results, this simulation used a tolerance level $T_{v}$ of $1 \%$.

During the simulation process, a certain number of difficulties had to be managed with the displacement of control points. An evident case to avoid is displacing control points leading to selfintersecting geometries. This is often the case when dealing with the sides. As a result, the geometries must be taken into account when defining the warping vector. This will be optimised in the future.

Another issue linked to the surfaces' parameterization occurred. A simple example highlighting this issue is to consider a scenario beginning with a flat surface. As it is being deformed, the control points' displacements will lead to some areas of the surface having a smaller density of control points than others. A solution would be to regularly re-parameterize the surface to avoid this. It would however add to the already huge computation time. This will be further studied in the future.

Anyhow, the simulation results were very close to those of the experiment for the values of vertical tool wear and roughness (table 4). Additionally, the tool's profile can be compared with the simulated one (figure 6). It appears that the simulation does not lead to the dissymmetry present in the experimentation. This can be due to several factors that haven't been included yet in the simulation, notably the influence of the dielectric flow and an eventual alignment error while machining the tool by wire EDM.

To validate fully the new simulation approach performance, further tests using a wider range of geometries are still required. However, these initial finding are encouraging and appear to demonstrate the viability of the method.

\section{Conclusions}

In order to overcome issues linked with the wear phenomenon in $\mu E D M$, it is important to be able to predict said wear. A viable method of simulation involving NURBS surfaces was presented. Although some discrepancies were found between experimental data and simulation results, those values remain acceptably close (deviation of $0.89 \%$ for the tool vertical wear and $6.09 \%$ for the roughness). As discussed, crater dimensions measurements were of paramount importance in the final results and are extremely important if a good accuracy was to be obtained. Another approach could be to use theoretical values for crater dimensions in lieu of the experimental ones. Efforts should also be put into preserving the surfaces' parameterization as well as reducing the computational times.

\section{Acknowledgements}

This work was supported by the Engineering and Physical Sciences Research Council [EP/F056745/1, EP/J004901/1].

\section{References}

[1] S. Dhanik, S. S. Joshi Modeling of a single resistance capacitance pulse discharge in micro-electro discharge machining J. Manuf. Sci. Eng., Trans. ASME, 2005, 127, pp.759-767.

[2] G. Bissacco, H. N. Hansen, G. Tristo, J. Valentinčič. Feasibility of wear compensation in micro EDM milling based on discharge counting and discharge population characterization. CIRP ann., 2011, 60 (1), pp.231-234.

[3] S. Bigot, A.Surleraux, G. Bissaco, J. Valenticic, A New Modelling Framework for Die-Sinking Micro EDM, Proceedings of the 9th International Conference on Multi-Material Manufacture. 2012, pp.51-55.

[4] J. Kennedy, R. Eberhart. Particle Swarm Optimization. Proceedings of IEEE International Conference on Neural Networks IV. pp.1942-1948.

[5] L. Piegl, W. Tiller. The NURBS Book. Springer, 1996.

[6] J.P. Pernot. Fully Free Form Deformation Features for Aesthetic and Engineering Designs, Unpublished doctoral dissertation, Università di Genova \& Institut National Polytechnique de Grenoble, 2004.

[7] S. Bigot, G. Bissacco, J. Valentinčič. Die-Sinking Micro EDM for Complex 3D Structuring - Research Directions. Int. Conf. on Multi-Material Micro Manufacture, Stuttgart, Germany, 8-9 November 2011. 